ÉGYPTE

monde arabe

\section{Égypte/Monde arabe}

25| 1996

Anthropologies de l'Égypte 2

\title{
Remarques sur les conditions d'une anthropologie du droit dans les sociétés du monde arabe et musulman
}

\section{Bernard Botiveau}

\section{CpenEdition}

Journals

Édition électronique

URL : https://journals.openedition.org/ema/825

DOI : 10.4000/ema. 825

ISSN : 2090-7273

Éditeur

CEDEJ - Centre d'études et de documentation économiques juridiques et sociales

Édition imprimée

Date de publication : 30 mars 1996

Pagination : 25-40

ISSN : 1110-5097

Référence électronique

Bernard Botiveau, «Remarques sur les conditions d'une anthropologie du droit dans les sociétés du monde arabe et musulman », Égypte/Monde arabe [En ligne], 25 | 1996, mis en ligne le 08 juillet 2008, consulté le 07 juillet 2022. URL: http://journals.openedition.org/ema/825; DOI : https://doi.org/ 10.4000/ema.825

Ce document a été généré automatiquement le 7 juillet 2022.

Tous droits réservés 


\title{
Remarques sur les conditions d'une anthropologie du droit dans les sociétés du monde arabe et musulman
}

\author{
Bernard Botiveau
}

1 Ces dernières années, les études sur le droit des sociétés arabes et musulmanes se sont beaucoup développées, quantitativement et qualitativement. Cette évolution participe d'un intérêt renouvelé, dans beaucoup de pays, pour les faits juridiques, perçus comme une des clés importantes de l'explication des dynamiques sociales. Comment rendre compte de la recherche sur le droit dans le monde arabe, notamment en Égypte, et selon quelles méthodes et procédures scientifiques une telle recherche est-elle amenée à se développer? Quelle part respective y prennent les écoles anthropologiques occidentales et les chercheurs des sociétés étudiées? Vers quelle répartition géographique ces recherches s'orientent-elles? Si les sociétés africaines ont tenu une place prépondérante dans ces recherches jusqu'à une époque récente, ce sont à présent les sociétés arabes et musulmanes qui semblent focaliser l'intérêt en raison, pour une bonne part, des changements introduits par l'action des mouvements islamistes et la question de l'application de la loi islamique. On observera tout d'abord que la participation de chercheurs issus de ces sociétés reste limitée, au sein d'une production où les recherches en langue anglaise - notamment celles produites dans le cadre des universités américaines - sont les plus nombreuses. C'est également le cas des études entreprises par l'anthropologie sociale et culturelle, en tenant compte toutefois d'une implication croissante des disciplines juridiques et de la science politique quand elles privilégient une approche du droit perçu comme un mode de représentation du monde.

2 Les remarques qui suivent n'ont pas pour objectif une présentation exhaustive de ces recherches, dont bon nombre semblent du reste en cours de réalisation, mais une mise en perspective de plusieurs manières d'étudier le phénomène juridique dans le monde arabe et musulman, et en particulier en Égypte. Les questions qui se posent au 
chercheur concernent la pluridisciplinarité, les lieux de recherche, les thèmes abordés et les méthodes suivies en relation avec l'histoire des disciplines ayant pris le droit, directement ou indirectement, comme objet d'interprétation des sociétés étudiées.

Nouveaux enjeux de l'anthropologie juridique du monde arabe et musulman

Tributaire des recherches ethnologiques effectuées depuis plus d'un siècle et apparues à l'ombre de l'impérialisme triomphant du xix siècle, comment l'anthropologie moderne peut-elle rendre compte des cultures juridiques du monde arabe et musulman actuel ? Pour les Morgan, Maine, Tylor et autres grands initiateurs de l'anthropologie sociale, coutumes et lois apparaissaient centrales dans le raisonnement ethnologique, sans être pour autant isolables et fonder une discipline autonome. La domination coloniale va cependant imposer l'idée de la nécessité d'analyser le phénomène juridique en tant que tel; il, faut connaître le droit pour pouvoir l'appliquer aux populations dominées, il faut connaître le droit musulman pour assurer un contrôle rationnel des populations musulmanes; d'où les recherches sur l'Adatrecht en Indonésie, sur le droit musulman et les droits coutumiers au Maghreb.

L'ethnographie juridique toutefois garde ses limites en se privant d'une dimension explicative globale, laquelle est abandonnée aux ethnologues "généralistes" qui intègrent le droit parmi d'autres variables. On rappellera par exemple que Marcel Mauss traitait des "phénomènes juridiques" parmi d'autres types de phénomènes sociaux (Mauss, 1967). Ces antécédents ont été fortement remis en cause par le processus de décolonisation qui rendait possible et nécessaire le renouvellement de l'anthropologie juridique. À condition de ne pas considérer le moment même de la décolonisation, dans la mesure où il marginalise les droits locaux et instrumentalise le droit pour en faire un droit national, mais le processus enclenché, qui va beaucoup plus loin et aboutit à ce sur quoi l'anthropologue du droit travaille aujourd'hui, à savoir l'existence d'univers différenciés qui ne se manifestent pas seulement hors d'Europe mais concernent l'ensemble des sociétés (Rouland, 1988; Arnaud, 1993). Cette conscience renouvelée, à travers des représentations variées de la modernité, que les faits sociaux sont inscrits dans une histoire, a grandement affecté le développement des analyses du phénomène juridique.

Différents facteurs y ont contribué. Le développement politique récent, tout d'abord, qu'il ne faut pas entendre ici dans un sens "développementaliste » réducteur, mais dans les modalités particulières de l'altération des institutions politiques, dont certaines sont antérieures au processus de décolonisation. Quoique l'on pense de l'efficacité ou même des chances de survie de l'État-nation au Moyen-Orient et ailleurs, on peut rechercher en quoi et selon quelles voies cette forme de pouvoir politique a produit du changement dans les sociétés arabes. En termes de modèles, l'Égypte représente un précédent dans le monde arabe, avec une réforme systématique des institutions et une codification du droit très élaborée. Les juristes y ont souvent été perçus comme les acteurs conscients d'un volontarisme politique qui visait à imposer une loi homogène et égalitaire, à l'image des avocats, souvent identifiés à l'élite politique (Reid, 1981; Hélin, 1995). Mais leur action sur le droit peut aussi être perçue comme une métaphore du changement social, comprenant le renouvellement conflictuel des anciennes générations de juristes, comme cela a été justement analysé à partir du développement des juridictions civiles en Turquie depuis un siècle (Starr, 1992). 
6 Ce qui rend aléatoire certaines approches anthropologiques actuelles - nous y reviendrons - c'est, sinon l'occultation, du moins une évidente sous-estimation de ce phénomène de modernisation institutionnelle, qui oblige à repenser le changement juridique dans des termes "stato-nationaux » sans pour autant ignorer qu'il ne rend pas compte de la totalité du phénomène juridique. L'exemple égyptien est là encore pour nous rappeler à quel point le développement politique a produit une culture juridique différente, fondée sur une mise en oeuvre assez poussée du principe d'universalité de la loi de l'État-nation. Les filières de formation, les écoles de droit - et à présent les écoles de la magistrature - ou la formation des juges dans le cadre de parquets, tous ces éléments ont eu des conséquences lourdes sur le fonctionnement de la justice, lieu central de l'affirmation du droit. De même que les degrés d'appel, les cours de cassation et les cours suprêmes sont là pour veiller à l'application d'un droit dont la rationalité postule l'homogénéisation des statuts. Ce sont ainsi des rôles nouveaux qui ont été définis dans le champ de l'activité juridique. Est-ce un phénomène surtout égyptien? Il faudrait citer d'autres expériences. Mais on observera des phénomènes similaires dans des pays comme le Yémen ou la Jordanie, réputés pour avoir conservé de fortes traditions de droits locaux.

7 Il ne s'agit pas ici d'une interprétation exclusive : le droit est le lieu de l'interaction et de la contradiction. Les acteurs en justice ont leurs stratégies, que leur permet un pluralisme normatif multipliant les voies de règlement des conflits. L'observation des conflits familiaux est à cet égard éclairante, dans la mesure où l'explication textuelle par le droit islamique ou les droits communautaires en général a longtemps régné, quasiment sans partage, sur l'ethnologie du droit. Les sociétés du monde arabe et musulman ont connu des changements considérables dans leur démographie, dans les structures familiales, sur lesquelles pesaient par ailleurs les politiques publiques, l'ouverture économique et l'essor des migrations de populations. Le recours des femmes aux tribunaux peut-il être analysé dans les seuls termes des réformes juridiques, dans la mesure où celles-ci tendaient au minimum à neutraliser les règles obsolètes du droit islamique, comme en Tunisie, ou à les confirmer, souvent explicitement, comme en Algérie et au Maroc?

8 Comme cela a été montré dans la cas d'une recherche comparée entre l'Iran et le Maroc (Mir-Hosseini, 1993), le modèle légal de la shari'a est de peu d'utilité pour rendre compte de certaines réalités sociales. Ainsi au Maroc où, pour différentes raisons (chômage, émigration des hommes ou modes d'insertion des femmes dans les réseaux de patronage), la capacité d'un nombre croissant de femmes à subvenir aux ressources du ménage bat en brèche une conception unilatérale de la famille patriarcale et, en tout cas, remet en cause le modèle légal de l'homme seul soutien de famille. Ce simple constat renvoie à une approche datée la représentation d'une « famille imaginaire » qui correspondrait aux canons du droit islamique classique (Bennani, 1993).

9 La situation différente des villes et des campagnes pourrait être invoquée pour contrarier la généralisation de ce type d'observations, mais encore faut-il des éléments de comparaison et l'on ne peut que s'étonner à ce propos du retard mis par l'ethnologie à envisager des études sur la famille en milieu urbain. On peut comprendre ainsi le constat d'une anthropologue étudiant une juridiction du statut personnel à Sanaa et notant que l'essentiel des études antérieures dans ce domaine ont été faites dans ce pays en milieu rural («Sana'a: Forgotten by Anthropology?»), en dépit d'un fort développement urbain du Yémen depuis au moins vingt ans, et alors même que les 
premiers éléments de cette enquête révèlent nombre de comportements "atypiques " du point de vue du droit islamique (Wurth, 1995). Dans le même sens, on peut mesurer le déplacement de perspective par rapport à une analyse textuelle, lorsqu'une étude sur le mariage temporaire en Iran montre comment cette institution classique est « instrumentalisée » par la politique, notamment pour tenir compte des déséquilibres démographiques causés par la guerre Iran-Irak (Haeri, 1989).

Ces mutations de la culture juridique prennent leur sens dans une évolution interne de ces sociétés, en relation avec des processus d'importation institutionnelle. Mais l'évolution des relations internationales devrait réorienter l'analyse du droit lorsque des systèmes juridiques subissent une altération rapide due à des recompositions régionales. Le Yémen offre là encore un très bon exemple de situation où les conditions de la recherche sur le droit ont été amenées à évoluer rapidement. Prenant leurs distances avec une anthropologie classique du droit (cf. Chelhod, 1972), différentes études ont attiré l'attention sur l'importance du renouveau urbain (Wurth, ibid.) et de l'effet "d'une politique législative centralisée sur la vie juridique locale (Mermier, 1989 ;Mundy, 1991a). Par ailleurs, les études traitant des problèmes posés par la codification du droit et l'émergence d'un nouveau type de juristes depuis la révolution de 1962 (Al-'Alimi, 1989; Al-MakhIafi, 1990; Botiveau, 1995), appellent un prolongement pour observer comment ces juristes interviennent dans la redéfinition du système juridique de l'État depuis l'unification du pays, tant sur le plan de l'application de la loi islamique - poussée par le Yémen du Nord - que sur celui de la confrontation entre le modèle «socialiste " du Sud et le modèle "conservateur » du Nord. Le droit foncier par exemple, largement abordé par l'ethnologie pour décrire des conflits locaux, l'est beaucoup moins dès qu'il s'agit de comprendre les logiques d'appropriation du sol en jeu dans les processus de territorialisation politique. Cela apparaît pourtant au Yémen dans la discussion sur le régime juridique de la propriété, collective ou individuelle. Dans un tout autre contexte, celui du Liban de l'après-guerre civile, on peut mesurer comment les juges intègrent à leur pratique une sensibilité particulière à l'environnement représenté par les relations syro-libanaises, lorsqu'ils s'interdisent, dans les affaires susceptibles de menacer l'unité de la justice, de « juger la guerre » (Bonne, 1995).

La Palestine offre un autre exemple du fait que l'international peut être, dans certaines situations, l'un des éléments importants, voire centraux de l'évolution du droit. Sur le foncier par exemple, l'utilisation abusive de normes islamiques et ottomanes (terres min notamment) par l'occupant israélien pour justifier la confiscation de terres appartenant à des Palestiniens, a été clairement montrée (Shehadeh, 1993). Le droit de la famille illustre aussi ce fait, quand on observe qu'un usage classique du droit islamique dans les tribunaux de Cisjordanie a été tempéré par la nécessaire composition avec le droit positif de l'État israélien (Layish, 1975); ou par l'insistance, dans les actions en justice intentées par des femmes, sur certaines règles du droit islamique (notamment le mahr/douaire), pour faire face aux conditions économiques précaires du statut de réfugié, comme cela a été observé à Jéricho (Antoun, 1980). La vie dans les camps de réfugiés a aussi produit des modes différents d'accès à la justice où, comme c'est le cas au Liban, des habitus nationaux se greffent sur des modes d'accès coutumiers associés au droit islamique (Peteet, 1987). Et c'est en fonction de ces nouvelles expériences juridiques que l'Autorité nationale palestinienne doit arbitrer en son sein les tentatives actuelles pour définir un droit national palestinien, entre les cultures juridiques adoptées par les Palestiniens de l'intérieur et de l'extérieur et, à 
l'intérieur, entre des expériences juridiques différentes, à Gaza, à Jérusalem ou dans d'autres villes de Cisjordanie.

L'analyse du droit dans le monde arabe et musulman devrait donc tenir compte des conditions réelles du développement juridique dans ces sociétés. Elles apparaissent, au quotidien, dans la confrontation avec la loi froide de l'État-nation comme, à un niveau global, dans la discussion des modèles de légitimation politique. Les recompositions sociales évoquées précédemment mettent en évidence le fait que les stratégies des individus et des groupes pour faire valider juridiquement leurs intérêts se traduisent par une grande variété de comportements, suivant une ligne courante qui est le développement d'un «droit de la pratique » destiné à «domestiquer les institutions de l'État moderne » au profit de ces intérêts (Le Roy, 1991). Mais on ne saurait, à mon sens, se limiter à cet aspect des usages du droit, si l'on admet que le droit est aussi le lieu d'une vaste confrontation politique qui prolonge les stratégies d'adaptation classique au pluralisme juridique par des stratégies de contestation, de conflit ouvert, dont on ne peut exclure qu'elles contribuent à imposer une définition non pluraliste de substitution au centralisme de l'État-nation, lequel est soupçonné de ne plus être en mesure de garantir une réelle citoyenneté. L'application de la sharîa est ici au centre des débats, et la compréhension de ce phénomène suppose une appréhension claire de ce qu'est le droit islamique et des débats qui ont cours à son propos dans l'anthropologie juridique.

Le droit islamique révélateur d'une crise de l'interprétation anthropologique du droit dans le monde arabe et musulman

13 La difficulté d'appréhender la place que prend le droit islamique dans le développement juridique moderne tient, me semble-t-il, dans la contradiction suivante. D'une part, un corpus normatif hérité du Coran, valorisé par la tradition prophétique et transmis par différentes traditions académiques, tient une place fort importante dans la mémoire des sociétés qui se réclament de l'islam. Ce corpus, enseigné aujourd'hui dans les universités islamiques, n'est pas simple affaire de spécialistes ; il fait sens pour beaucoup de gens : le projet islamiste de renouer avec l'application de la shari'a affirme en effet que les sociétés musulmanes ont été dépossédées de leur "mémoire islamique ", tout en s'appuyant sur elle pour rendre crédible son programme. D'un autre côté, tout en prenant acte de ce réfèrent, le chercheur ne peut qu'être frappé de son ambiguïté et de l'incertitude régnant quant à son contenu comme aux modalités selon lesquelles il intervient dans la réalité des sociétés contemporaines. Le cas le plus fréquent est celui dans lequel un processus de codification a écarté du droit positif la majorité des normes se trouvant dans le Coran, ne maintenant sa présence que dans le droit de la famille et sous une forme codifiée selon les règles constitutionnelles de l'État-nation, donc rendant difficilement identifiables comme islamiques les normes coraniques maintenues, si ce n'est comme des «sources » du droit parmi d'autres.

Le "paradigme égyptien », qui s'est diffusé dans la plupart des pays du Machrek et de façon moins explicité, mais tout aussi efficace, dans les législations du Maghreb, rend compte de cette situation. Dans ce système, le droit est interprété et appliqué par des juristes formés dans une logique stato-nationale où prédomine l'illusion que la même loi est appliquée à tous et dans une langue juridique accessible au citoyen ordinaire. Une autre situation est celle de l'Arabie Saoudite, où le droit islamique est cerise régir là totalité des relations sociales, alors qu'il est permis de douter non seulement de l'adéquation de ce droit à là complexité sociale, mais encore de la fidélité de ses 
interprètes officiels aux doctrines développées par nombre d'ulémas dans le passé. Par exemple, rien n'indique que le «droit pénal islamique » n'y soit utilisé autrement que pour justifier une répression civile et politique indispensable à la survie de la monarchie régnante, c'est-à-dire sans les garanties établies par les jurisconsultes classiques; de même que le système bancaire « islamique » semble avoir pour fonction principale dé légitimer la gestion de la richesse au profit d'une élite affairiste liée au pouvoir politique.

Des distorsions tout aussi importantes entre idéal islamique et réalités sociales ont été relevées dans des cas tels que le Soudan, le Pakistan et l'Iran, lorsque la politique impose une conformité à la shari'a (Mayer, 1991). Transplanté dans les pays européens qui ont pris en considération les cultures juridiques des populations migrantes, le droit islamique peut être représenté, de façon non métaphorique, comme une source indirecte, ou au moins un réfèrent incontournable du droit, français par exemple (Deprez, 1988 ; Botiveau, 1991).

16 Autrement dit, si l'on peut historiquement identifier une culture juridique islamique faite de normes et de valeurs contenues dans la loi coranique, ainsi que d'un savoir et de techniques (la jurisprudence) destinés à traduire en termes socialement acceptables le droit (haqq), il est risqué de parler abstraitement d'un droit islamique en général, en le privant de l'historicité à laquelle il peut prétendre. Car dans les sociétés actuelles, il n'existe pas de droit positif correspondant au droit islamique, mais des droits positifs validés par des États et tenant compte bien entendu de cette culture juridique islamique sans en être totalement dépendants; droits autorisant des stratégies variées qui permettent aux individus et groupes de s'adapter et de servir leurs intérêts.

Pourtant, l'idéalisation du droit islamique est perceptible dans les représentations communes comme dans les constructions scientifiques. Il serait sûrement commode de voir là une prédisposition à l'essentialisme qui caractériserait, peu ou prou, tout débat ayant trait à des normes sociales, des valeurs que l'on peut historiquement situer par rapport à une référence religieuse, en l'occurrence le Coran. Mais cela ne rendrait compte que partiellement des enjeux de cette discussion. Il est clair que les études orientales (islamologie, ethnologie juridique, droit comparé, histoire...) ont contribué à répandre des représentations essentialistes selon lesquelles le droit islamique serait marqué définitivement par sa genèse de droit religieux, qu'il serait inapte au changement, irréductible aux autres cultures; et ce pour différentes raisons et à des degrés très variables, faut-il y insister, selon les auteurs : influence de l'évolutionnisme, ethnocentrisme, faible distance à l'égard du discours dogmatique des ulémas, dont le savoir orientaliste dépend pour ses sources. Mais cela ne suffit pas à expliquer certaines orientations actuelles, alors que les «orientalistes '» eux-mêmes ont largement reconsidéré certaines de leurs analyses en fonction du développement des Étatsnations dans le monde arabe et musulman (par exemple, Schacht, 1959; Linant de Bellefonds, 1955 ; Milliot, 1953), et en tenant compte de certaines études ethnologiques antérieures, surtout françaises au Maghreb (Maunier, 1932 ; Berque, 1944).

L'anthropologie juridique contemporaine est, certes, au cœur de ces débats, mais l'explication est sans doute plus subtile. Il est intéressant à cet égard de rappeler les discussions provoquées par le livre de Lawrence Rosen, Anthropology of Justice (1989). Anthropologue réputé, Rosen a aussi été avocat et il utilise sa connaissance pratique du champ juridique au service d'une construction théorique qui décrit le droit islamique comme l'expression d'une réalité culturelle beaucoup plus large, celle du mode de vie 
marocain. Ayant longuement fréquenté les juridictions marocaines où sont traités les conflits familiaux, et surtout une juridiction de Sefrou, dans le Moyen Atlas marocain, ayant pu établir quelques comparaisons avec des juridictions tunisiennes et indonésiennes, Rosen propose, au-delà de l'exemple de Sefrou, ce qu'on peut appeler un idéal-type - dans la mesure où il se réfère explicitement à Weber - du qâdi et de la juridiction islamique. Séduisante parfois, et souvent juste lorsqu'il analyse des mécanismes de fonctionnement de la justice qu'on peut retrouver dans beaucoup de systèmes différents (indétermination du droit, négociation), présentant suffisamment d'informations sur les débats judiciaires même s'il n'a livré qu'une faible partie des cas qu'il a observés, sa démonstration appelle cependant de sérieuses réserves, qui ont été fort justement émises par plusieurs recensions de l'ouvrage (Mundy, 1991,Layish, 1994,0ssman, 1991).

La principale réserve, qui ouvre la voie à d'autres questions, porte sur la relation entre le juge de Sefrou et l'institution judiciaire marocaine, à laquelle il est organiquement lié. Comment comprendre que ce juge exerce son office dans une autonomie quasiidéale, dans une sorte d'« autarcie judiciaire », bien loin de la hiérarchie judiciaire de Rabat ? Il est de ce point de vue significatif que la référence permanente soit faite non à la section «statut personnel» du tribunal de première instance de Sefrou, que le juge semble présider, mais à la juridiction du qâdi de Sefrou (" the court of the qadi of Sefrou »). Comment ce juge prend-il des décisions pour lesquelles on pourrait le supposer lié par la Mudawwana, le code du statut personnel marocain? Comment interviennent la hiérarchie judiciaire, le parquet, les avocats ? Rosen n'élude pas ces questions, puisqu'il consacre un chapitre du livre au problème de la relation entre le juge et l'État et aux dérogations interprétatives à la Mudawwana. Mais c'est en fait pour minimiser la portée des interventions extérieures dans la juridiction du qâdi. Ainsi, s'il reconnaît que ces interventions ont eu pour résultat un affaiblissement de l'autonomie qu'il postule face au pouvoir central, cela n'est que relatif; par exemple, la faible participation des avocats dans ce système (Sefrou) tiendrait en partie au fait que les plaideurs utilisent volontiers d'autres intermédiaires locaux par crainte de dépenses excessives, ce qui est plausible, mais surtout au fait que la plupart des juges préfèrent s'adresser directement aux plaideurs, explication moins convaincante. Quoiqu'il en soit, l'une des raisons avancées de cette autonomie serait que le gouvernement marocain ne s'immisce guère dans les affaires judiciaires ni n'a beaucoup instrumentalisé le droit, lequel reste à l'écart de la politique. Cela tiendrait au caractère "individualiste " de la société marocaine, dont le pouvoir politique se doit de tenir compte; or, si cette explication culturaliste, récurrente dans la démonstration, sert le propos, elle appelle d'autres questions. Que représente le droit s'il reste isolé des débats sur le droit, comme ceux, récents, sur la Mudawwana (Al-Ahnaf, 1994) .ou sur les droits de l'homme (Ossman, ibid.)?

20 Au total, beaucoup d'incertitudes. On relèvera à ce propos le parallèle fait par Martha Mundy (ibid., 1991b) entre l'ethnologie juridique berquienne, ancrée dans une connaissance pratique et théorique de l'islam scripturaire marocain (cf. également les textes de Berque présentés dans Mahé, 1996), et la démarche de Rosen, plus extérieure, si l'on en juge par un faible emprunt aux sources écrites islamiques, comme si le procès judiciaire dépendait avant tout de procédures orales. Pourtant, l'importance de la dynamique scripturaire au Maroc a été soulignée de longue date (Berque, 1944); et l'on 
peut se demander si le rapport à cette source ne s'inscrit pas, comme pour les législations modernes, dans une opposition trop systématique entre écrit et oral.

Cependant, la logique de ces critiques ne devrait pas conduire, à mon sens, à masquer l'observation des modalités selon lesquelles la négociation (qui n'est pas l'inverse, ni l'alternative exclusive de l'imposition du droit) opère dans la juridiction observée, qui n'est en réalité pas si « islamique » que cela est annoncé. On peut observer à cet égard que la «négociation » judiciaire, traditionnellement non envisagée par les juristes occidentaux, peut devenir une des clés interprétatives de la Justice contemporaine, en Europe ou ailleurs, même si l'on admet que le respect du droit écrit implique que tout n'est pas négociable en justice. Les nouveaux rôles du juge en Europe par exemple, peuvent être pris pour les symptômes "d'une mutation profonde de la rationalité moderne $»^{1}$.

II reste que, en dépit de la Justesse de certaines observations, Rosen ne fait que suggérer la comparaison, en mentionnant que la «juridiction du qâdi » pourrait apprendre beaucoup aux juristes occidentaux. C'est même la conception d'une institution spécifique, propre à un espace culturel (" a typical Islamic court », p. 6) qui prévaut. À cela, un ouvrage récent a répondu, concentrant le débat sur l'utilisation des données historiques dans l'anthropologie du droit et jaugeant les inconvénients d'une application trop mécanique de la théorie weberienne à l'anthropologie de la justice. Il s'agit de l'ouvrage de Haim Gerber, State, Society and Law in Islam (1994), dont le soustitre, Ottoman Law in Comparative Perspective, précise les enjeux de la discussion. Je ne reviens sur ce livre, recensé ailleurs (Botiveau, 1995; Pierce, 1995), que pour en souligner les apports à la discussion et à la critique des approches néo-weberiennes de l'islam en général, et de celle de Rosen en particulier. Gerber conteste principalement deux points dans l'analyse weberienne: le fait que la juridiction islamique soit représentée comme une source d'arbitraire et d'imprévisibilité, alors que, notamment, la sécurité des échanges marchands requiert au contraire une loi appliquée avec constance et donc prévisible; et l'arbitraire présupposé du pouvoir ottoman, attribué à cette variante du patrimonialisme qu'est le sultanisme.

Même si l'on peut localement (par exemple à Sefrou) observer des pratiques judiciaires qui confirment le poids de la culture islamique, la généralisation est critiquable et Gerber estime même que l'Empire ottoman échappe dans son ensemble à cette analyse. Gerber cependant se situe lui aussi dans une perspective weberienne, au moins par son insistance sur la bureaucratie ottomane. Par ailleurs, on peut regretter également la généralisation du propos, dans la mesure où l'on pourrait trouver bien des "Sefrou » dans l'Empire ottoman, tout comme des cas sans doute très différents.

Quoiqu'il en soit, la démonstration rappelle que l'anthropologie juridique aurait tort de garder ses distances à l'égard des recherches historiques, qui montrent que, sur le long terme, des variations importantes sont observables quand il ne s'agit pas de contreexemples. On peut contester l'analyse selon laquelle la négociation du droit se faisait principalement, entre les forces politiques, en amont des juridictions, à l'occasion du prononcé des fetvas (fatwa) par le ShaykhuI Islam à Istanbul. Elle existe aussi à des échelons inférieurs. En revanche, certains faits devraient être mis en évidence, ne serait-ce que parce qu'ils démontrent que des vérités très largement partagées ne sont pas nécessairement fondées, Le cas sans doute le plus caractéristique, dégagé par Gerber de ses recherches dans les archives de Busra au XVII ${ }^{e}$ siècle, est celui de l'interdiction du ribâ selon le «droit islamique ». On sait combien d'hypothèses sont 
aujourd'hui basées sur cette donnée (cf. les nombreuses études et monographies ayant trait à «l'économie islamique »), légalement fondée mais sociologiquement discutable. Ainsi, l'interdiction du prêt usuraire est le plus souvent présentée aujourd'hui comme une norme incontournable d'un point de vue « islamique». Or, Gerber nous rappelle fort utilement que, dans l'Empire ottoman, cette norme n'a pas été simplement "contournée" par des ruses (hila) solution qui permettait aux orientalistes de confirmer l'antériorité, dans la vie quotidienne, d'une norme immuable et non susceptible de transgression explicite, mais que des fatwa donnèrent aussi un point de vue islamique tout à fait officiel, à savoir que l'interdiction du ribâ ne correspondait pas aux intérêts du marché et qu'il n'y avait donc pas lieu d'en tenir compte.

Le juridique et le politique : l'exemple des études sur la justice

25 Je voudrais, sous ce titre, apporter quelques remarques supplémentaires en guise de conclusion, tenant aux potentialités que représentent actuellement les études sur la justice et spécialement sur la pratique du juge, étant donné la proximité de cet acteur avec le pouvoir politique quel que soit son statut dans la hiérarchie judiciaire et indépendamment de sa situation dans la société. La distance prise avec une tradition anthropologique idéalisant les rôles et les statuts, ou réduisant à des « isolats » des faits sociaux et culturels historiquement construits, devrait permettre à une anthropologie $\mathrm{du}$ droit renouvelée de se situer dans un contexte où intervient aussi, dans le développement historique, le politique ; il s'agirait également de resituer le politique "sur son véritable terrain, non plus celui des institutions formelles, mais celui des actions qui visent le maintien ou ta modification de l'ordre établi » (Balandier, 1978). La dynamique islamiste et le thème de l'application de la shari'a ont largement contribué à accréditer l'idée que, parmi les institutions politiques, les institutions judiciaires étaient l'un des lieux où les conflits politiques étaient susceptibles de se négocier.

Mais avant de revenir sur ce point, rappelons que cette avancée a été rendue possible par le constat, auquel l'anthropologie du droit semble à présent largement acquise, de la nécessité d'historiciser l'étude des pratiques judiciaires. Un exemple récent de cela, en ce qui concerne la culture juridique islamique, est la réunion en 1994, à Amsterdam et Leiden, de la Joseph Schacht Conférence on Theory and Practice of Islamic Law ${ }^{2}$, dont l'objectif était d'établir un état des lieux des disciplines concourant à la connaissance du droit des sociétés arabes et musulmanes. Un nombre conséquent des quelque 40 communications présentées, portant sur des périodes et des lieux fort différents du monde arabe et musulman, ont montré la difficulté, voire l'impossibilité de réinvestir tel quel l'idéal type du qâdi dans l'analyse des pratiques judiciaires, contemporaines ou non. Ce constat n'était pas toujours explicite mais il ressortait autant d'une relecture, par les historiens présents, de l'histoire des disciplines impliquées et des conditions auxquelles l'analyse des processus discursifs peut se révéler aujourd'hui pertinente (cf. à ce propos Messick, 1993), que du besoin des « contemporanéistes » de se repérer dans leurs terrains de recherche respectifs, en les situant par rapport aux aléas du développement historique. Une rencontre internationale de ce type renouvelle donc la réflexion sur la recherche en contestant des analyses trop dépendantes d'un orientalisme qui a longtemps régi ce champ de recherche. On peut, d'un autre côté, se demander si l'étude du droit islamique aujourd'hui diversifie suffisamment les thèmes traités. Une majorité des études présentées privilégiaient en effet quelques thèmes comme le système des waqf et le droit de la famille, en renouvelant toutefois les références utilisées, à l'image de la fatwa qui tend à relayer l'analyse textuelle (tradition prophétique ou traités de fiqh) en introduisant un élément de référence aléatoire parce 
que directement relié à une dynamique sociale. De fait, le statut renouvelé de la fatwa dans les sociétés actuelles montre l'intérêt d'accentuer et de diversifier l'accès à cette source juridique (voir par exemple Paradelle, 1995\}.

C'est peut-être, comme nous le rappelions ci-dessus, parce qu'un droit islamique idéal est introuvable, qu'il convient de repérer dans quels contextes sociaux la culture juridique islamique intervient pour moduler des débats déclinés sur le mode de la norme islamique. Les pratiques judiciaires contemporaines ouvrent ici de nouvelles perspectives quand te rôle du juge-fonctionnaire de l'État est fortement questionné par l'obligation, réaffirmée avec solennité par la Constitution, de se conformer à la loi islamique, comme c'est le cas .actuellement en Égypte. On constate par exemple qu'en quelques années, l'approche de cette question par le juge égyptien a sensiblement évolué, comme le confirment les recherches menées par Baudouin Dupret, notamment sur l'accès, non plus médiatisé mais direct, aux sources du figh classique (Dupret, 1994 et 1995), alors qu'on sait aussi que le milieu judiciaire, en dépit de sa différenciation, reste marqué par le moule de l'État-nation (Abécassis \& Ardisson, 1995). Le fait que le juge contemporain tente de se réapproprier certains référents du qâdi autorise-t-il pour autant à faire de son discours un discours situé culturellement, c'est-à-dire dépendant de ce droit islamique idéal, alors que tout porte à constater que les discours judiciaires liés à l'application de la shari'a sont l'expression d'enjeux politiques liés à la légitimation des États actuels (Ferrié \& Radi, 1995)?

D'autres observations devraient être ajoutées, par exemple pour ce qui relève du «normal» (juridictions ordinaires) et de l'« exceptionnel» (juridictions spéciales). Si les recherches passant par le politique - au demeurant peu nombreuses actuellement sont amenées à se multiplier, est-il cependant logique d'envisager une division du travail de l'anthropologie appliquée au droit et à la justice, laissant aux juristes et aux politologues le soin de développer une anthropologie juridique normative (Legal anthropology, et aux anthropologues celui de s'investir dans une anthropology of law centrée sur les expressions juridiques des cultures (Mir Hosseini, ibid., préface) ? Les expressions contemporaines $\mathrm{du}$ phénomène juridique dans le monde arabe et musulman appellent, certes, une diversification des terrains de recherche, des problématiques et des accès disciplinaires sans laquelle la différenciation de la vie juridique risquerait d'être occultée, mais la complémentarité de ces recherches n'empêche pas leur corrélation étroite, si l'on admet que l'anthropologie en général, et celle du droit en particulier, visent à chercher un sens aux comportements humains dans différents types de relations en société.

\section{BIBLIOGRAPHIE}

Abecassis F., Ardisson D., «Les magistrats égyptiens : variations sur le modèle de l'État », Droit et Cultures $n^{\circ} 30,1995$, p. 17-45.

Al-Ahnaf M., « Maroc. Le Code du statut personnel », Monde arabe/Maghreb-Machrek, n¹45, 1994, p.3-26. 
Al-'Alimi R. M., Al-taqlîdiyya wa-l-hadâtba fi-l-nizàm al-qânûni al-yamani. Dirâsa muqârana (Tradition et modernité dans le système juridique yéménite. Étude comparée), Université de Sanaa, Faculté des lettres, 1989.

Al-Makhlafi M. A. A., « Min târikh al-tashrî' fi-l-yaman » (Sur l'histoire de la législation au Yémen), Dirâsât yamaniya, 21,1990.

Antoun R. T., " The Islamic Court, the Islamic Judge and the Accommodation ofTraditions; a Jordanian Case Study », IJMES, 12, 1980, p. 455-467.

Arnaud A. J., « Anthropologie juridique », in Arnaud (A. J. et al.), Dictionnaire encyclopédique de théorie et de sociologie du droit ( $2^{\mathrm{e}}$ éd.), Paris, LGDJ, 1993.

Balandier G., Anthropologie politique (3éd.), PUF, Paris, 1978.

Bennani F., La division du travail entre les époux à la lumière du droit marocain et du fiqh islamique (en arabe), Faculté des sciences juridiques, économiques et sociales, Marrakech, 1993.

Berque J., Essai sur la méthode juridique maghrébine, M. Leforestier, Rabat, 1944.

Bonne E., « La magistrature libanaise à l'épreuve des reconstructions de l'État d'après-guerre ", Droit et Cultures, $\mathrm{n}^{\circ} 30,1995$, p. 107-121.

Botiveau B., «Islamic Law in the French Légal Context », Cambridge Anthropology, 16-2, 1992, p. 85-96.

- "Yémen ; politiques législatives et mutations de la culture Juridique », Chroniques yéménites, CFEY, Sanaa, 1992.

- Compte rendu de Gerber (H.) in Bulletin critique des annales islamologiques, 1994.

Chelhod J., « La société yéménite et le droit », L'homme, 1972-2, p. 67-86.

Deprez J., Droit international privé et conflits de civilisation, La Haye, Académie de droit international, vol. 211,1988.

Dupret B. « Entre le droit et la loi. Le juge et la normalisation islamique du droit positif », Droit et Cultures, $n^{\circ} 30-2,1995$, p. 47-64.

- « À propos de la constitutionnalité de la sharî'a. Présentation et traduction de l'arrêt du 26 mars 1994 de la Haute Cour constitutionnelle égyptienne ", Islam, Law and Society (à paraître).

Ferrié J.-N. et Radi S., " L'aporie d'Al-'Ashmawy et les limites de l'anthropologie relativiste : est-il possible de décrire comme un fait culturel la position de deux cadis sur l'application de la sharî'a? », Droit et Cultures n 30,1995, p. 65-76.

Garapon A., « La question du Juge », Pouvoirs n 74, (Les juges), 1995, p. 13-26.

Gerber H., State, Sociêy and Law in Islam. Ottoman Law in Comparative Perspective, State Univ. of New York Press, Albany, 1994.

Haeri S., Law of Désire. Temporary Marriage in Iran, I. B. Tauris, Londres, 1989.

Helin E., « La profession d'avocat en Tunisie », Correspondances n 36,1995, p. 10-15.

Layish A., Women and Islamic Law in a Non-MusIim State. A Study Based on Decisions of the Shari'a Courts in Israël, Israël Univ. Press, Jérusalem, 1975.

-Compte rendu de Rosen (L), 1989, in Istamic Law and Society, vol. 1-1,1994, p. 139-140.

Le Roy E., « Les usages politiques du droit », Les Afriques politiques, Coulon Ch. et Martin D. C., La Découverte, Paris, 1991, p. 109-122. 
Linant de Bellefonds Y., «Immutabilité du droit musulman et réformes législatives en Égypte », Rev. int. de droit comparé, 1955, p. 5-39.

Mahé A., «Jacques Berque et l'anthropologie juridique du Maghreb », à paraître dans Droit et Cultures $n^{\circ} 33$.

Maunier R., Loi française et coutume indigène en Algérie, Domat-Montchrestien, Paris, 1932.

Mauss M., Manuel d'ethnographie, Payot, Paris, 1967.

Mayer A. E., Islam and Human Rights, Westview Press, Boulder \& San Francisco, 1991.

Mermier F., « Des artisans face aux importateurs ou l'ange maudit du souk », Peuples méditerranéens $\mathrm{n}^{\circ}$ 46,1989, p. 155-164.

Messick B., The Calligraphic State: Textual Domination and History in a Muslim Society, Univ. of Califomia Press, Berkeley, 1993.

Milliot L. (éd.), Travaux de la semaine internationale de droit musulman (Paris, 2-7 juillet 1951), Sirey, Paris, 1953.

Mir-Hosseini Z., Marriage on Trial. A Study of Islamic Law. Iran and Morocco Compared, I.B. Tauris, Londres, 1993.

Mundy M., Compte rendu de Rosen (L), 1989, History Workshop Journal, 32, 1991, p. 184-192.

-, Legal Traditions in a Cecentralized Polity: Islamic Law in a Locality of Noryh Yemen in the 1970s ", comm. présentée au colloque Normes Juridiques et pratiques sociales dans le monde musulman, EHESS, Paris, Janvier 1991.

Ossman S., Compte rendu de Rosen (L.), 1989, Annuaire de l'Afrique du Nord, 1991.

Paradelle M., « Entre mufti et juge : la place du religieux dans l'organisation judiciaire égyptienne (à partir d'une lecture de l'article 381 du code de procédure pénale) », Droit et Cultures, $\mathrm{n}^{\circ} 30$, 1995, p. 77-89.

Pierce L., Compte rendu de Gerber (H.), 1994, Islamic, Law and Society, vol. II-3, 1995, p. 365-371.

Peteet J., « La Justice au quotidien dans les camps palestiniens au Liban », Rev. d'études palestiniennes $\mathrm{n}^{\circ} 24,1987$, p. 25-39.

Reid D. M., Lawyers and Politics in theArab Worid, 1880-1960, Bibiiotheca Islamica, Minneapolis, Chicago, 1981.

- Cairo University and the Making of Modem Egypt, Univ. Press, Cambridge, 1990.

Rosen L., The Anthropology of Justice. Law as Culture in Islamic Society, Cambridge University Press, Cambridge, 1989.

Rouland N., Anthropologie juridique, PUF, Paris, 1988.

Schacht J., «Islamic Law in Contemporary States », The American Journal of Comparative Law, vol. Vlll-1959, 2, 1959, p. 133-147.

Shehadeh R., The Law and the Land. Settlements and Land Issues under Israeli Military Occupation, Passia, Jerusalem, 1993.

Starr J., Law as Metaphor. From Islamic Courts to the Palace of Justice, State University of New-York Press, New-York, 1992.

Wurth A., " A Sana'a Court: The Family and the Ability to Negociate ", Islamic Law and Society, vol. $2, \mathrm{n}^{\circ} 3,1995$, p. 320-340. 


\section{NOTES}

1. Voir Garapon, citant De Munck J., Lenoble J. et Molitor M., L'avenir de la concertation en Europe, Centre de philosophie du droit, Université catholique de Louvain-la-Neuve, 1995 : « Le juge met en œuvre une conception plus pragmatique de la raison, a qui suppose l'instauration d'un dialogue en situation avec tous les acteurs concernés par la norme discutée ». Cette appréciation de Garapon pourrait être rapprochée de celle de Rosen («It appears that the central goal of the qadi is to put people back into a position of negociating their own ties, within the bounds of the permissible. » (p. 65. Voir référence dans la bibliographie)

2. Rijksuniversiteit Leiden \& Universiteit van Amsterdam, 6-10 octobre 1994. Une première série des communications présentées, concernant le droit familial, a été publiée dans Islamic Law and Society, vol. 11-3,1995.

INDEX

Mots-clés : islam, anthropologie, droit

\section{AUTEUR}

BERNARD BOTIVEAU

CNRS-Iremam 